\title{
LÉGISLATION
}

\section{Les Droits d'Enregistrement des Concessions de Distribution d'Énergie à l'origine, au moment des cessions, et au sujet du rachat.}

\author{
Par Paul BOUGaUlt, Anocal à la Cour d’Appel de Lyon.
}

\begin{abstract}
L'auteur examine' les questions d'enregistrement annoncées dans le titre et, pour plus de clarté, al divise son étude en un nombre de chapitres ćgal à celuí des principaux poinls à iraiter.
\end{abstract}

\section{PREMIERE PARTIE}

Principes de l'enregistrement au Débu'T

Les contrats de concessions municipales de distribution d'énergie sont des actes administratifs qui doivent être enregistrés dans les 20 jours de l'approbation préfectorale.

Ce délai est indiqué par l'article 78 de la loi du 15 mai 1818 ainsi conçu : " demeurent assujettis au timbre et à l'enregistre"menl sur la minute, dans le délai de 20 jours, conformément aux "lois existantes : les actes des autorités adıninistratives et des " établissements publics portant transmission de propriété, "d'usufruit et de jouissance; les adjudications ou marchés de " toute nature aux enchères, au rabais, ou sur soumission ; les " cautionnements relatifs à ces actes. "Le délai ne court que du jour où le maire remet à la partie qui doit effectuer la formalité de l'enregistrement, l'arrêté du Préfet, conformément à la Circulaire du Ministre de l'Intérieur du 6 septembre 1853, remise qui sera constatée par une déclaration du Maire, datée et signée en marge de l'arrêté (décision du Ministre des Finances du 22 janvier 1855 , Dal. 1856.3. 56,rapportée daus le Code de l'Enregistrement, sous l'article 20 de la loi du 22 frimaire, an VII).

Si l'article 78 de la loi du 15 mai 1818 est relatif au délai, et, par conséquent, doit être cité à ce titre, la question du tarif (pour les concessions municipales) est toujours réglée par l'article $51, n^{\circ} 3$, de la loi du 28 avril 1816 (1) qui est ainsi conçu • "les " adjudications au rabais et marchés pour constructions, répara" tions, entretien, approvisionnements et fournilures dont le " prix doit être payé par les administrations locales ou par des "établissements publics seront sujets au droit de 1 franc par " 100 francs. "A ce tarif il faut ajouter deux décimes et demi, c'est-à-dire 25 centimes.

D'un arrêt de la Cour de cassation, toutes chambres réunies, en date du 12 juillet 1875 (affaire Société Petit, Dal. 1875 I.341), il résulte que les mots " autorités administratives " comprennent les communes parce qu'ils sont extrêmement larges, et que, d'autre part, le mot "marché " comporte tous les contrats passés par elles quels qu'en soient l'objet et la forme. Nous faisous toutefois remarquer, pour qu'il n'y ait pas de confusion, que ce mot ne

(1) Nous visons la loi de 1816, parce que l'article est relatif aux concessions municipales. Si le prix était payé par l'Etat - - ce cui peut avoir une importance trés considérable pour les concessions d'Etal; - il y aurait lieu de remarquer que le tarif de 1816 n'esl conservé par l'arlicle 78 de la loi de 1818 que pour les administrations autres que celle de l'Etat (d'où le not "demeurent " assujettis employé par ledit article) ; en ce qui concerne les Administrations de l'Etat, les textes sont' nombreux : le tarif a été un droit fixe, d'abord, pour devenir un droit proportionnel de $0,25 \%$; il est de $1 \%$ sans décime depuis la loi du 29 juin 1918 (artiele 15). saurait englober les emprunts passés par les communes, opérations purement financières qui sont dispensées d'enregistrement en vertu de l'article 80 de la loi du 15 mai 1818 ainsi conçu : " tous les actes, arrêtés et décisions des autorités administratives, " non dénommés dans l'article 78 , sont exempts du timbre sur " la minute, et de l'enregistrement, tant sur la minute que sur "l'expédition. Toutefois, aucune expédition ne pourra être " délivrée aux parties que sur papier timbré ."

Comment doit se faire l'application de ce tarif ?

Plusieurs questions sont à examiner :

a) Il faudra d'abord se demander si l'on se trouve en présence d'une convention devant être analysée, et considérée comme contenant des dispositions indépendantes les unes des autres, ou si, au contraire, on se trouve en présence d'un acte absolument homogène, ne devant être enregistré qu'au tarif unique. Sur cette question délicate je ne puis que renvoyer le lecteur aux considérations fort longues que j'ai données dans mon Commentaire du Cahier des Charges type, notamment au Numéro 106 dans lequel j'ai examiné le principe, au No 107 dans lequel j'ai indiqué que la règle générale vieut qu'en matière de concession la présomption soit en faveur de l'homogénéité, et au No 108 qui contient des exemples de dispositions indépendantes relevées dans la pratique.

b) En supposant l'absence de conditions indépendantes, on devra prendre tout d'abord la question normale, celle qui se présente le plus souvent, c'est-à-dire la réunion dans un même contrat de trois éléments : le privilège exclusif, une fourniture faite à l'autorité concédante, ef l'indication absolue que les travaux effectués par le concessionnaire resteront à cette autorité.

c) On doit envisager, au moins à titre d'impression, ce qui doit se passer quand ces trois éléments ne se trouvent pas réunis.

Sur ces deux dernières questions, à cause de leur importance, nous allons donner des décisions destinées à faciliter la formalité.

\section{I. - Enregistrement normal : privilège, fournitures, travaux.}

La question, sur ce point, ne peut faire de doute : le tarif de $1,25 \%$ doit être appliqué au total de ces deux éléments :

10 Le prix représentant les fournitures que la commune s'engage à prendre, étant admis obligatoirement que, faute d'une stipulation, ce prix doit être calculé d'après le maximum.

$2^{\circ}$ Le montant (déterminé par une évaluation faite par le concessionnaire) des travaux devant rester à la ville à la fin de la concession.

Ces questions ont été vivement discutées en matière de conces- 
sion de distribution d'ean (1) et l'on peut dre que tous les dét ails ont été exuminés. Lin arrèt qui, à ce titre,nous paraît déjà bien complet est celui de la Chambre Civile du 5 février 1889 (1)al. 1889, I.198), Compagnie des Eamx contre Enregistrement) dans lequel nous voyons une Compagnie investie du privilège exclusif de la distribution des eaux pendant 65 ans dans la ville d'Elbeuf, assurée de loucher une rederance de $8.800 \mathrm{fr}$. par an pour fournitures à la Ville, mais devant adandonner à celle-ci : " tous les ouvrages "d'adduction et de distribution, tous établissements et acces" soires se rattachant à la conduite des eaux, en bon étal "d'entretien sans indemnité. "La Cour déclare que le droit de $1,25 \%$ doit ètre pris is la fois sur le montant du marché portant sur l'eau (annuités de 8.800 francs cumulées) et sur l'épalualion des travaux ; sur ce dernier point qui paraît avoir été le seul vivement discule devant elle, la Cour proclame : "Il ressort " manifestement des termes du traité du 30 décembre 1881 et du " cahier des charges $y^{\prime}$ annexé que le prix lotal du marché ne " consiste pas seulement dans la redevance annuelle de $8.800 \mathrm{fr}$. " payée pour la fourniture de l'eau, mais aussi dans l'avantage "que procure à la Compaṣie le privilçge exclusif pendant “65 ans de conduire et de distribuer les eaux, au moven de " conduites placées dans le sol des rues ; la portion du prix que " représente ce privilège exclusif n'étant pas exprimée, le droit " ne peut être liquidé que sur l'évaluation faite par les parties “ de l'importance des ouvrages dont l'exécution est ainsi rému" nérée. "Il faut retenir de cette phrase que, d'après la Cour, la valeur du privilège exclusif de ce que l'on appelle communément mais inexactement le "monopole", se traduit par le montant des travaux à effectuer.

(1) On entend quelquefois dire qu'entre la distribution d'eau et celle du courant électrique, il existe une différence d'appréciation pour le fisc : dans la première, les Tribunaux regarderaient d'une façon spéciale le volume de l'ean cédée et attribueraient, au contrat, le caractère de vente d'une chose mobilière (tarif de $2^{\circ}{ }_{0}$ autrefóis, de $5 \%$ aujourd'hui) ; dans la seconde, ils considéreraient que l'on ne vend pas de Kilowatts, mais que le fournisseur promet de diriger son usine et de produire du courant : l'ouvrage et le travail du producteur étant plus particulièrement pris en considération.

En réalité, cette prétendue distinction n'est que le résultat d'une confusion. Les différents arrêts qui sont relatés dans cet article sur la distribution des eaux, ont bien été rendus dans de véritables entreprises de distribution et, comme on le voit, les Tribunaux admettent parfaitement le contrat de louage d'ouvrage ou d'industrie et le tarif de $1,25 \%$ sur la totalité des marchés et de l'évaluation des ouvrages; car le trait caractéristique de l'entreprise de distribution, e'est de commander un travail considérable.

Si la Cour a effectivement considéré le volume d'eau comme une chose principale faisant l'objet du contrat qui alors est une vente, c'est que l'on se trouvait en présence plutôt d'une police que de toute autre convention.

Ainsi l'arrêt du 26 novembre 1873 (Dal. 1874. 1. 217) était relatif à la prestation d'un volume d'eau par la Société Dufour à la maison Nationale de Charenton : la Société avait vivement réclamé l'application du bail de choses, c'est-à-dire le droit de 20 centimes par 100 francs d'après le tarif de l'époque. On peut encore citer l'arrêt du 20 novembre 1880 (Ch. Civ. Dal. 1881. 1. 169, Ville de Dijon contre Enregistrement) ; cet arrêt analyse la convention par laquelle la Ville, sur l'excédent de l'eau de la source du Rozier destinée aux fontaines publiques, avait promis des concessions individuelles à des particuliers ; il déclare qu'un contrat de cette nature ne constitue ni un bail (car l'objet considéré se détruit par l'usage) ni un contrat de louage d'ouvrage (les travaux étant des moyens d'exécution, par conséquent de simples accessoires de la convention dont l'eau est l'objet principal) : il faut appliquer le tarif de vente d'une chose mobilière (loi de Frimaire an VII, article 69 , paragraphe 5, $N^{\circ} 1$ ). On remarquera que la Ville a été conclamnée à payer le tarif de $2 \%$ parce que c'est elle qui reçoit le prix de l'eau, et non pas le tarif de $1 \%$ de l'article 51 , No 3 de la loi du 28 avril 1816 qui est applicable quand la ville acquitte ellemême le prix de la fourniture.
Mais, un arret plus complet encore est celui de la Chambre Civile du 20) mai 1890 (1)al. 1890. I. 349, affaire Compagnic générale des Eaux de Saint-Nazaire contre Enregistrement) dans lequel les données étaienl identiquement les mèmes. I a Cour, terme pour terme, reproduil l'arrè précédent sur la nécessité de comprendre dans la perception le monlant des travaux deslinés à l'Administration a $47.000 \mathrm{fr}$., sauf augmentation ou dimimution d’après le devis qui pourrait ètre présenté au Receveur et elle base encore cette obligation sur le privilege exclusif dont la valeur est représentée par celle des travaux et des ouvrages. Mais, devant elle, on avait aussi discuté le montant du marché de fournitures et fait valoir ce qui suit : lo marche ne constılue pas un bloc absolu ; un article prévoit certaines interruptions possibles dans le service; un autre déclare que le prix du mètre cube est de 15 centimes au maximum, mais par définition, 111 "maximum " est toujours susceptible de baisser. La Cour rèpond : "l'obligation de fournir n'en est pas moins, pour la Compagnie, " une obligation actuelle dont l'exécution mlterieure n'a pas "d'influence sur la fixation du droit ; en outre, la réduction " éventuelle du prix n'empèche pas qu'il ne soit exprimé dans " l'acte et qu'il ne fournisse la base d'une détermination certaine " de son montant total; qu'il n'y a done pas lieu pour les parties " de faire une déclaration estimative de la fourniture sur d'autres " bases.

Il est évident que la mème jurisprudence s’applique aux contrats de concession pour une listribution d'électricité ou de gaz (voir Rép. Prat. de Dalloz Vo Enregistrement No 925). A l'appui de cette affirmation le hèpertoire Pratique de Dalloz cite : décision du Tribunal de Langres 7 mai 1890 (Rép. Pér. Enregistrement $N^{0} 7.441$ ) ; Trib. Màcon, 12 mars 1901 (Rev. Enregistrement 2.778) ; Trib. de Béziers, 5 décembre 1906 (Journal Enregistrement 27.339 ) ; Tribunal d'Arhois, 27 juin 1907 (Rev. Enregistrement 1.412) ; (h. des Req., 25 lévrier 1908 (Dal. 1908, 1.159).

\section{II. - Enregistrement d'un traité incomplet.}

Il se présente dans certains cas qu'un des éléments ci-dessus indiqués vient à manquer ; et c'est cette éventualité que nous allons examiner :

a) Il peut, notamment, ne pas exister de fourniture réellement promise à la Ville par le concessionnaire; c'est-à-dire d'aprc̀s le contrat lü-même, on peut voir persister une indépendance absolue entre le concessionnaire et le concédant, la commune ne s'engageant, en aucune facon, à prendre quoi que ce soit comme fourniture; dans ce cas, _- c'est l'évidence même, — l'évaluation ne saurait ètre faite; car, pour qu'il y ait perception fiscale, il faut qu'un accord au moins de principe se révèle et, dexplus, la comptabilité publique étant ouverte aux agents fiscaux, si jamais il y a une police postérieure qui intervient, l'Administration percevra sans difficulté le droit légalement dù, sans aucunc súpercherie possible.

Mais, l'absence d'évaluation n'est légale que si ladite indépendance existe réellement; au contraire, si la convention montre, d'ores et déjà, le concédant et le concessionnaire en relations directes, par exemple "s'il est dit que le concessionnaire desservira la commune pour ses besoins ou que la commune prendra tout ce qui lui est nécessaire "il doit y avoir évaluation immédiate, tant pour les fournitures gratuites que pour les fournitures futures (et, en ce qui concerne celles-ci, à titre hypothétique) ; on peut consulter sur ce point l'arrêt du 7 décembre 1898 (Ch. des Req. I)al. 1899. I. 471, affaire Compagnie générale des Eaux contre Administration de l'Enregistrement). Dans celle 
aflaires des foumilures élaient failes à la Municipalité de Pantin, ful moment de la passation du contrat, en vertu d'unc convention antérieure, et le Tribunal avait admis que les fournitures fulures etaicnt acquises à la Compagnie, parce que le privilège exclusif Clail domné et interdisait à la commune de s'adresser ailleurs ; il y a eu une évaluation à plus de 700.000 rrancs, à titre provisoire; la Chambre des Requetes approuve le Tribunal d'avoir dit. que les droits seront perçus sur cette évaluation émanant du fisc, à moins que la Compagnie souscrive dans les délais impartis une déclaration rectificative.

Nous nous résumerons donc sur ce point en disant : sil y a indépendance absolue au sujel des fournitures, poinl d'évaluation ; si, an contrairc, il y a relalion de consommateurs à rlients, l'evaluation sera obligatoire ; le Répertoire pratique de Dalloz No 925 envisageant des hypothèses dans lesquelles il n'y avait probablement que des fournitures à prendré comme élément taxable, indique ce qui suil : " la valeur du monopole reste " en dehors de la liquidation du droil de marché à moins que " l'Administration ne puisse déterminer cette valeur, comme dans " dans le cas où l'entrepreneur doit faire des fournitures gratuites " à la Ville et où ces fournitures doivent être évaluées au prix le plus favorable résultant du traité (Tribunal de Vassy, 3 mars "1909, Rev. Enregistrement 4.911) ; mais la circonstance que la "Ville bénéficierait d'un tarif cle faveur pour l'eau ou l'éclairage " lestiné à ses services publics ne saurait être considérée comme " une charge à ajouter au prix : Tribunal Arbois, 27 juin 1907 " (Rev. Enregistrement 4.412); Tribunal de la Seine, 6 mai 1908 " (Hev. Enregistrement 4.652) ; Tribunal de Marseille, 28 jan" vier 1910 (Rev. Enregistrement 5.319); pour faire décider le " contraire, il. faudrail que la réduction du prix atteignît une " proportion très élevée, Tribunal de Corbeil, 11 février 1892 (Rev. de lEnregistrement 81).

b) D'un autre cité, il peut n'anoir iamais été stipulé que les travaux, en fiu le concession resteraient gratuitement à l'autorité concédante ; cela, notamment, pent se produire dans d'anciens contrats, dans lesquels il étail dit qu'arrivée au terme prévu, la commune rachèterait, à dire d'expert, les travaux effectués; cela peut encore se préseuter avec le cahier type, si on a donné une rédaction appropriée à l'article 22 ; on sait que ledit article permet à la commune d'attendre la fin du contrat, pour dire si elle accepte gratuitement les travaux et ourrages ou bien si elle ne les prend point et préfère les voir enlever par le concessionnaire à ses frais. Si, au moment de la rédaction du contrat, la commune opte d'une manière définitive pour l'acceptation des travaux, elle n'a qu'à ne pas accepter d'écrire le paragraphe en italique que contient l'article 22.

I a question reste embarrassante si, comme cela se produit souvent, le cahier des charges conserve jusqu'à la fin pour la commune le droit de choisir. Certains receveurs ont une tendance à considérer, au point de vue liscal, que l'évaluation des travaux est, en quelque sorte, la rançon du privilège exclusif, et ils voudraient percevoir le droit de 1,25\% sur l'évaluation des travaux, par le seul fait qu'il y aurait " monopole stipulé ", alors mème que lesdits travaux pourraient ne pas rester à la ville; ils citent les arrèts précités en maliòre d'eau, sans faire attention que lesdits Iravaux, précisément daus les arrèts intervenus, devaient Loujours ètre la propriété de la commune concédante. A l'encontre de cette théorie, on peul faire la remarque suivante : la Cour de Cassation a rendu un arrèt extrêmement intéressant (Ch. des Req. 25 février 1908, affaire Sociélé Méridionale contre Administration de l'Enregistrement, 1)al. 1908, I. 519). Il faut conslater tout d'abord que la Chambre des Requètes a été très allïmative sur la nature de l'hypothèse qui lui était soumise; en toutes lettres, elle constale qu'il y a un véritable monopole dans la convention intervenue en 1902 entre la commune de Quarante el la Société, qui avait pour cause déterminante de procurer l'éclairage et la force électriques à ladite commune; que chacune des parties a stipulé à son profit des prérogatives particulières se. traduisant pour la Société en un véritable monopole de vente el que, si la commune, propriétaire d'ouvrages de canalisalion et de certains appareils électriques. en a permis l'usage à la Société, à charge par elle de les entretenir en bon état, et a autorisé l'établissement de nouvelles canalisations sur le domaine public et le domaine communal, cette clause, dans l'esprit des parties, n'est qu'accessoire et qu'un moyen d'effectuer les travaux nécessaires pour la mise en cuvre de l'entreprise.

Ainsi, dans cette affaire, il y avait bien monopole, et d'autre part, il y avait, si l'on se rapporte au jugement, bien des détails qui, à la rigueur, si l'on admet les théories extensives de certains lieceveurs, pourraient tous être considérés comme des éléments de taxation : la commune de Quarante avait stipulé des fournitures à son profit ; elle avait imposé à la Société l'entretien du matériel communal dont elle lui abandonnait la jouissance ; enfin, la Société avait le droit de construire des ouvrages nouveaux.

Or, le jugement est extrèmement explicite sur la question des évaluations à faire : il déclare textuellement : " attendu qu'en matière de marchés ne comportant pas la stipulation d'un prix payable à l'entrepreneur, le droit de $1 \%$ est perçu sur l'évaluation que les parties sont tenues de fournir des objets mohiliers qui en sont susceptibles; que, dans le cas présent, les seuls objets pouvant ètre évalués sont les fournitures à faire où travaux à exécuter ; qu'en conséquence les droits doivent être calculés : $1^{0}$ d'après la valeur de l'éclairage de 120 lampes de 16 bougies dont la Société doit, dès le début, assurer le fonctionnement, sans prëjudice des droits supplémentaires en cas d'augmentation ; $2^{\circ}$ sur les frais d'entretien du matériel appartenant à la commune dont la charge lui incombait et qu'elle a imposés à la Société."

Si l'on remarque que le jugement, dans cette analyse, est muet sur la question précise des travaux que la Société pouvait effectuer sur le domaine municipal ; si l'on remarque, d'un autre côté, que, dans le contrat, il n'était dit nulle part, que la commune devait entrer en pleine propriété gratuitement, des travaux nouveaux, on est bien obligé de convenir que, même lorsqu'il y a monopole, l'ouvrage qui n'est pas abandonné gratuitement par le concessiomnaire à l'autorité concédante, n'est pas un élément de taxation.

\section{DEUXIEME PARTIE}

\section{ENREGISTREMENT DE IA CESSION DES CONCHSSIONS}

Nous avons à nous préoccuper dans cette partie d'une concession existante, mème en plein fonctionnement, et qui doit être cédée par son titulaire à un tiers, avec l'approbation du Conseil municipal. (Voir dans mon livre précité le Commentaire de l'article 33 sur ce point.)

On doit distinguer deux hypothèses, essentiellement différentes bien que, dans le public, on ait une certaine tendance à les confondre

Ou bien la cession est gratuite, ou bien elle est faite du prix d'argent.

A) La cession gratuite s'analyse en réalité en une simple subslitution d'un concessionnaire nouveau au concessionnaire initial. Or, il a été reconnu que cette modification n'entraîne pas un marché nouveau et par conséquent doit ètre enregistrée à un simple droit fixe. Ce principe est, depuis longtemps, acceple 
même par l'Administration pour une soumission qui n'a d'autre effel que de mețre un particulior à la place de l'adjudicataire initial. sans spécifier un prix nouveau, ni aucune modification nouvelle : et il en est de même pour l'arrèté municipal qui l'a approuvée et par conséquent u'a fait qu'adhérer à la substitution des personnes, sans rien innover quant aux conditions premières et quant aux prix; il en est ainsi, mème si le cessionnaire obtient un supplément de prix du maitre du marchć. La simple augmentation de l'obligation n'entraine pas, en effet, novation de la dette primitive. Il ne serait dû à raison de cette clause qu'un droit proportionnel sur l'augmentation du prix stipulé. (Voir Dictionnaire des Rédacteurs Vo Marchés No 346.$)$

Du reste, l'Administration quand il n'y a pas de prix stipulé est vraiment tolérante ; même elle ne réclame pas le droit de cession de créance, c'est-à-dire le tarif non modifié de $1 \%$ sur l'engagement d'un concessionnaire nouveau de rembourser à l'ancien son cautionnement, si l'acte de cession de marché ne lui est présenté que comme acte administratif et à raison de l'agrèment de l'autorité administrative à la substitution du premier titulaire.

Enfin. il est de principe que lentrepreneur entrant ne doil aucun droit en raison des travaux que Inj laisse l'entrepreneur sortant.

B) La cession à litre onéreux est au contraire, d'après les conceptions formelles du fisc, soumise au tarif qui frappe le marché portant sur les choses mobilières. Yais il ne faut pas qu'il $v$ ait d’équivoque : le Larif n'est appliqué qu'à la somme qui représente le prix de la cession, les avantages reçus par le cédant du cessionnaire, sans englober le moins du monde les conditions initiales acceptées par le premier titulaire, vis-à-vis de l'autorité concédante (eodem verbo, No 3.18 ). C'est en effet une affaire yui se traite entre deux personnes et l'autorité n'intervient que pour donner son consentement de principe.

Quant au prix de la cession, il est considéré comme soumis au tarif qui régit la transmission des valeurs mobilières ( $2 \%$ autrefois, $5 \%$ aujourd'hui).

En effet, l'article 69, paragraphe $5, n^{\circ} 1$ de la loi de Frimaire an VII soumet à l'enregistrement : les adjudications, ventes, reventes, cessions, rétrocessions, marchés, traités et tous autres actes translatifs de propriété à titre onéreux de meubles, ou objets mobiliers généralement quelconques. Et la concession, prise dans son ensemble, constitue un droit mobilier de jouissance et d'exploitation. Sans doute, on pourrait dire que l'article 51, No 3 de la loi du 28 avril 1816 réduit au tarif de $1,25 \%$ le droil exigible "sur les marchés, pour constructions, réparations, " entretien, approvisionnements et fournitures dont le prix " doit ètre payé par les administrations locales ou les établisse" ments publics ". Mais c'est une faveur faite pour faciliter lesdites administrations francaises et leur procurer des contractants à meilleur comple (voir Cassation Ch. civ. 7 mai 1882, Dal. 1882. 1.425, aflaire Chardenet et Crédit lyonnais contre Enregistrement). Elle n'a donc rien à voir avec la cession à laquelle deux particuliers sont seuls intéressés (voir aussi Ch.' des
Req. 19 janvier 1909, Dal. 1912, 1.363, ville de L,yon contre Enregistrement) (1).

Sans doute, on peut se demander si l'Administration dont on connaît les laulastiques prélentions ne pourrail pas (au lieu d'admettre la ression d'un droit mobilier assujelli au tarif de $2 \%$ autrefois en vertu de l'arlicle 69, paragraphe 5 , No 1 , ol aujourd'hui de $5 \%$ en vertu de l'article 24 de la loi du 25 juin $1920)$ soutenir un des systèmes suivants et dire :

$1^{\circ}$ Que les canalisations placées dans le domaine public sont considérées comme des immeubles par nature (puisqu'elle a su faire admettre par la Cour de Cassation, en matière d'octroi, qu’elles peuvent être considérées comme des matériaux employés dans des constructions immobilières). Sur ce point, on peut, au contraire, répondre que la Régie prend l'opération de cession dans son ensemble, c'est-ì-dire' comme constituant pour le toul la transmission d'une chose mobilière (voir solution du 25 janvier 1890, Rép. Pér. de l'Enregistrement No 8993), et il faut se réjouir, car le tarif immobilier (article 69, paragraphe $7, N^{\circ} 1$ de la loi de Frimaire an VII) qui était avan la loi du 25 juin 1920 de $7 \%$ a passé, par l'efret de l'article 25 de cette dernière loi, à $10 \%$.

20 Que la cession du matériel el des approvisionnements de l'entreprise constitue entre l'entrepreneur entrant et l'entrepreneur sortant une cession de fonds de commérce. Il est vrai qu'aujourd'hui avec l'enregistrement obligatoire dans les mêmes délais et l'identité des tarifs, la question n'aurail plus la même importance qu'autrefois. Toutefois, on a Loujours adinis sur ce point malgré une solution contraire du Tribunal Civil de Bordeáux du 7 août 1889 (Kép. Pér. Enregistrement No 7 456 ), qu'il ne saurait en être ainsi : car le cessionnaire tient son privilège de la ville et non pas du cédant. Il n'y aurait vente de'fonds de commerce que dans le cas où, en annexe à la concession elle-mème, le cédant aurait organisé des industries et des commerces particuliers, par exemple, pour la vente des compteurs, du coke et du goudrou (Rép. Prat. Dalloz Vo Enregistrement No 930) (1).

(1) Cet arrêt que nous retrouverons plus loin au sujet de la rétrocession et du droit dû pour les approvisionnements, notamment, a fait dans la référence précitée, l'objet d'une note très inressante dans laquelle il est spécifié que quand les poursuites de la Régie ont pour but de demander une déclaration estimative, la discussion est toujours réservée sur la sommè elle-même après fixation par les autorités administratives.

(2) En réalité, la théorie parait être la suivantè : quand on détache d'une exploitation une concession, pour la céder, il n'y a pas cession d'un fonds de commerce ni même d'un élément dı fonds, car les éléments qui sont exclus sont, avec les créancés et les dettes, les traités divers qui créent des relations personnelles entre le propriétaire du fonds et des tiers; il a été jugé cependant, mais en matiève de nantissement, que la concession d'une distribution d'énergie formait l'élément fondamental du fonds de commerce du concessionnaire qui était propriétaire d'une usine électrique et (qu'elle était comprise dans le nantissement (Gaz. Pal., 6 juillet 1913, Cour de Toulouse du 30 avril 1913). Mais cette décision est vivement critiquée : Rép. Prat. Dal. Vo Fonds de Commerce $n^{\circ} 15$ et 341 .

(A suivre.) 